\title{
Maximum mass-loss rates of line-driven winds of massive stars: The effect of rotation and an application to $\eta$ Carinae
}

\author{
C. Aerts ${ }^{1}$, H. J. G. L. M. Lamers ${ }^{2,3}$, and G. Molenberghs ${ }^{4}$ \\ 1 Institute of Astronomy, Catholic University of Leuven, Celestijnenlaan 200 B, 3001 Leuven, Belgium \\ 2 Astronomical Institute, Utrecht University, PO Box 80000, 3508 TA Utrecht, The Netherlands \\ 3 SRON Laboratory, for Space Research, Sorbonnelaan 2, 3584 CA Utrecht, The Netherlands \\ ${ }^{4}$ Center for Statistics, Limburgs Universitair Centrum, Universitaire Campus, Building D, 3590 Diepenbeek, Belgium
}

Received 31 March 2003 / Accepted 10 January 2004

\begin{abstract}
We investigate the effect of rotation on the maximum mass-loss rate due to an optically-thin radiatively-driven wind according to a formalism which takes into account the possible presence of any instability at the base of the wind that might increase the mass-loss rate. We include the Von Zeipel effect and the oblateness of the star in our calculations. We determine the maximum surface-integrated mass that can be lost from a star by line driving as a function of rotation for a number of relevant stellar models of massive OB stars with luminosities in the range of $5.0<\log \left(L / L_{\odot}\right)<6.0$. We also determine the corresponding maximum loss of angular momentum. We find that rotation increases the maximum mass-loss rate by a moderate factor for stars far from the Eddington limit as long as the ratio of equatorial to critical velocity remains below 0.7. For higher ratios, however, the temperature, flux and Eddington factor distributions change considerably over the stellar surface such that extreme mass loss is induced. Stars close to the Eddington-Gamma limit suffer extreme mass loss already for a low equatorial rotation velocity. We compare the maximum mass-loss rates as a function of rotation velocity with other predicted relations available in the literature which do not take into account possible instabilities at the stellar surface and we find that the inclusion thereof leads to extreme mass loss at much lower rotation rates. We present a scaling law to predict maximum mass-loss rates. Finally, we provide a mass-loss model for the LBV $\eta$ Carinae that is able to explain the large observed current mass-loss rate of $\sim 10^{-3} M_{\odot} \mathrm{yr}^{-1}$ but that leads to too low wind velocities compared to those derived from observations.
\end{abstract}

Key words. stars: early-type - stars: mass-loss - stars: winds, outflows - stars: evolution - methods: statistical stars: individual: $\eta$ Car

\section{Introduction}

Rapidly rotating massive stars may suffer mass-loss rates that are considerably higher than those of non-rotating stars. Stellar evolution calculations indeed show that rapidly rotating stars must lose a large fraction of their mass in a relatively short time (Langer 1998). For the calculation of the evolution of rotating stars Langer (1998) used an expression for the mass-loss rate of rotating stars, based on an interpolation and extrapolation of the models from Friend \& Abbott's (1986) early study of rotating line-driven winds. This expression predicts that the mass-loss rate becomes extremely high when the star rotates close to critical velocity. This result was criticized by Glatzel (1998) and by Owocki et al. (1998), who find that the mass-loss rates at critical velocity do not differ too much from those of a non-rotating star.

Maeder \& Meynet (2000) propose a new scaling law for the increase of mass loss with the rotation velocity by taking into account the Von Zeipel effect and the oblateness of the star.

Send offprint requests to: $\mathrm{C}$. Aerts, e-mail: conny@ster.kuleuven.ac.be
This law depends on the Eddington factor $\Gamma$, the CAK lineforce parameter $\alpha$ (Castor et al. 1975, hereafter called CAK) and the critical velocity. It predicts a finite mass-loss rate close to critical velocity for stars sufficiently far from the Eddington limit, i.e. for $\Gamma$ roughly below 0.3 . For higher values of $\Gamma$, however, extreme mass loss is derived. Our current study elaborates further on this by including any kind of instability at the base of the stellar wind that might increase the mass-loss rate. In particular, the purpose of our work is to investigate how much mass can maximally be expelled from a rotating star by radiation pressure due to spectral lines, by allowing for the most optimistic circumstances near the sonic point. This work can be seen as a generalisation of Maeder \& Meynet (2000). We explicitly integrate the momentum equation for different latitudes to find the surface-integrated maximum mass-loss as a function of rotation velocity rather than using a scaling law for the mass loss of a non-rotating and a rotating star. Moreover, we allow for an unspecified extra force (e.g. due to pulsation or magnetic fields) at the stellar surface that changes the velocity gradient at the sonic point and helps in this way in the onset of the mass loss. 
Very recently, Aerts \& Lamers (2003, hereafter termed Paper I) presented a formalism to derive maximum equatorial mass-loss rates $\dot{M}_{\max }^{\mathrm{eq}}$ of massive stars for which the wind is driven by radiation pressure in spectral lines with finite disk correction, photon tiring and rotation included. The latter, however, was included only for the equatorial region. The rotationinduced decrease of the radiative flux from the poles to the equator, called gravity darkening, as well as the oblateness of the star, was neglected. Aerts \& Lamers solved the momentum equation for different positive values of the velocity gradient at the sonic point as boundary condition. They then selected the highest possible mass loss for which an outflowing wind is still obtained. Such a formalism is the most flexible one to see how much mass can be driven by a CAK-type radiative force on spectral lines, because an unspecified possible extra force (such as due to stellar oscillations, magnetic fields or instabilities) at the base of the wind is allowed for.

These maximum mass-loss rates were compared with those predicted by classical self-regulating CAK wind theory and with the models including multiple scattering (Vink et al. 2000, 2001) which do not include instabilities at the base of the wind. The result of Paper I was that line driving can induce a maximum equatorial mass loss that is at most a factor 2-3 higher than the one of a self-regulated line-driven wind, in agreement with the earlier results of mass-overloaded wind solutions found in the studies by e.g. Poe et al. (1990), Owocki et al. (1994), Gayley (2000) and Feldmeier \& Shlosman (2002). In this paper we investigate to what extent the Von Zeipel effect and rotational flattening changes the results obtained in Paper I, by performing the calculations for an oblate star and providing total surface-integrated maximum mass-loss rates instead of only equatorial ones. Our results can be compared with those of Pelupessy et al. (2000), who calculated line-driven mass-loss rates of rotating hot stars with oblateness, the von Zeipel effect and the proper finite disk correction factor taken into account, but who did not allow for the presence of an extra force.

The concept of the maximum mass-loss rate was introduced in Paper I because mechanisms other than radiation pressure and rotation are common among massive stars and may influence their mass loss. This will affect the evolution of the star if the resulting mass-loss rate is much higher than for selfregulated line-driven winds. As Paper I revealed the occurrence of maximum mass-loss rates which are only moderately higher than those considered up to now for (super)giants, and in view of the intense debate about the role of rotation on mass loss of massive stars (see e.g. Langer 1998; Glatzel 1998; Owocki et al. 1998; Maeder \& Meynet 2000), we devote the current paper to the detailed study of the dependence of the maximum mass-loss rates, determined with the formalism of Paper I, on rotation.

The paper is organised as follows. In Sect. 2 we briefly recall the ingredients of the formalism and the definitions used in Paper I to derive the maximum mass-loss rates. In Sect. 3 we subsequently present the maximum mass-loss rates as a function of the rotation velocity for a grid of stellar models of massive stars. We do this by outward integration of the momentum equation for different latitudes and by summing the corresponding local mass-loss rates for all stellar latitudes. We also compare our values for the surface-integrated maximum mass loss with those found from Langer's (1998) empirical relation and with Maeder \& Meynet's (2000) scaling law. We also determine the maximum loss of angular momentum. We provide a maximum mass-loss recipe derived from multiple regression of the calculated grid, i.e. for the range $5 \leq \log \left(L / L_{\odot}\right) \leq 6$ in Sect. 4. An application of our mass-loss calculations to the luminous blue variable $\eta$ Carinae is presented in Sect. 5 . We end with a discussion in Sect. 6.

\section{Maximum mass-loss rates: The formalism}

The starting point for the formalism developed in Paper I was to understand to what extent the conservation of momentum and energy allow the occurrence of extremely high mass loss. To do this, we adopted the most flexible conditions possible, i.e. we allowed for an unspecified force at the base of the stellar wind to help drive the wind. Such a force can result from stellar oscillations or a magnetic field or any instability, i.e. phenomena that are common in massive stars but that are not commonly included in line-driven wind calculations yet. Under this condition, which replaces the usual singularity and regularity condition adopted in a self-regulating wind, it was investigated how large $\dot{M}_{\text {eq }}$ can become such that the momentum equation remains solvable for a CAK-type parametrisation of the line-driving. This was done in the approximation of a uniformly rotating star for which the deviation from spherical symmetry of the physical stellar quantities and of the wind was neglected. Finite disk correction and photon tiring were taken into account, as well as a wind temperature law $T(r) \sim r^{-n}$ with $n=1$. It was shown in Paper I that the resulting mass loss depends only very weakly on $n$.

The momentum equation was solved numerically from an assumed position of the sonic point outwards, for increasing values of the mass-loss rate, until the amount of wind material had increased so much that the acting forces could no longer make it escape from the star. This condition is expressed mathematically by allowing any positive velocity gradient at the sonic point so that there is outflow. Since we are interested in the maximum mass-loss rate that can be lifted out of the potential well of the star by radiation pressure on spectral lines, we did not solve the subsonic structure of the wind. The velocity gradient at the assumed sonic point was calculated numerically from the momentum equation. We refer to Sect. 2 of Paper I for a detailed description of the theory we adopt here. In Paper I we allowed the radius of the sonic point, $R_{\mathrm{S}}$, to be situated in the interval $[1.01,1.1] R_{\star}$. It was shown there that the highest equatorial mass-loss rates were obtained for a sonic point as close as possible to the stellar surface, a well-known result from classical wind theory. We hence fixed the sonic point at the position $R_{\mathrm{S}}=1.01 R_{\star}$ for all results shown in this paper.

In Paper I, we selected the maximum mass-loss rate in the equatorial plane, termed $\dot{M}_{\max }^{\text {eq }}$, out of all the physically relevant solutions of the momentum equation for which this equation still has an outflowing wind. The maximum velocity that occurs in the wind was defined as $v_{\max }$. It was shown in Paper I that the $v_{\max }$-values corresponding to $\dot{M}_{\max }^{\mathrm{eq}}$ are typically a few hundred $\mathrm{km} \mathrm{s}^{-1}$ for all considered cases. This is much lower 
Table 1. Stellar parameters of the 9 non-rotating models for which the maximum mass-loss rates were determined in Paper I. The line force parameters $\alpha$ and $k$ are taken from Pauldrach et al. (1986). The critical velocity $v_{\text {crit }}$ is expressed in $\mathrm{km} \mathrm{s}^{-1}$ while $\sigma_{\mathrm{e}}$ is expressed in $\mathrm{cm}^{2} \mathrm{~g}^{-1}$. The mass-loss rate is given in units of $10^{-6} M_{\odot} \mathrm{yr}^{-1}$.

\begin{tabular}{ccccccclllr}
\hline \hline Model & $\log L / L_{\odot}$ & $T_{\text {eff }}$ & $M\left(M_{\odot}\right)$ & $R\left(R_{\odot}\right)$ & $\Gamma$ & $v_{\text {crit }}$ & $\alpha$ & $k$ & $\sigma_{\mathrm{e}}$ & $\dot{M}_{\max }^{\text {eq }}$ \\
\hline Model 1 & 6.0 & $50000 \mathrm{~K}$ & 60 & 15 & 0.434 & 873 & 0.64 & 0.124 & 0.34 & 4.3 \\
Model 2 & 6.0 & $40000 \mathrm{~K}$ & 50 & 23 & 0.521 & 644 & 0.64 & 0.124 & 0.34 & 6.1 \\
Model 3 & 6.0 & $30000 \mathrm{~K}$ & 40 & 42 & 0.613 & 426 & 0.59 & 0.17 & 0.32 & 11.8 \\
Model 4 & 5.5 & $40000 \mathrm{~K}$ & 40 & 10 & 0.209 & 873 & 0.64 & 0.124 & 0.34 & 0.8 \\
Model 5 & 5.5 & $30000 \mathrm{~K}$ & 35 & 18 & 0.221 & 609 & 0.59 & 0.17 & 0.32 & 1.0 \\
Model 6 & 5.5 & $20000 \mathrm{~K}$ & 30 & 40 & 0.250 & 378 & 0.565 & 0.32 & 0.31 & 3.4 \\
Model 7 & 5.0 & $40000 \mathrm{~K}$ & 30 & 8 & 0.087 & 846 & 0.64 & 0.124 & 0.34 & 0.1 \\
Model 8 & 5.0 & $30000 \mathrm{~K}$ & 25 & 14 & 0.089 & 584 & 0.59 & 0.17 & 0.32 & 0.2 \\
Model 9 & 5.0 & $20000 \mathrm{~K}$ & 20 & 32 & 0.119 & 345 & 0.565 & 0.32 & 0.31 & 0.5 \\
\hline
\end{tabular}

than the values of the terminal velocity $v_{\infty}$ predicted by the classical or modified CAK-models. It was also found in Paper I that the mass-overloaded solutions at a high percentage $(>80 \%)$ of the critical rotation velocity have kinked velocity laws, in agreement with earlier studies in the literature. In the following section we focus entirely on the mass-loss rates so we do not list explicitly the corresponding wind velocities, which are all of the order of a few hundred $\mathrm{km} \mathrm{s}^{-1}$.

\section{The effect of rotation on the maximum mass-loss rates}

We describe the mass-loss rates and angular momentum loss for stellar models that are rotationally flattened and gravitydarkened. The (equatorial) stellar parameters of the considered models are listed in Table 1 . They span a range of $20000<$ $T_{\text {eff }}<50000 \mathrm{~K}$, at high luminosities between $10^{5}$ and $10^{6} L_{\odot}$.

In Table 4 of Paper I, the equatorial maximum mass-loss rates for 9 spherical stars of different stellar parameters and for a large range of equatorial velocities, ranging from zero rotation up to the critical velocity, was presented, assuming the stars to be spherical solid rotators. The radiative acceleration was calculated for a spherical star with a homogeneously illuminated stellar disk, i.e. without gravity darkening. Under these simplifying assumptions the maximum mass-loss rates of stars rotating near critical were found to increase only with a factor 2-3 compared to those of non-rotating stars.

In reality, the radius, effective temperature, luminosity and Eddington factor differ for different values of the co-latitute $\theta$ due to the rotation of the star (we use the convention that the pole of the star corresponds with $\theta=0^{\circ}$ ). To investigate the correct effect of rotation on the overall maximum mass loss, the procedure outlined in Sect. 2 has to be repeated for each co-latitude $\theta$ and for increasing values of the equatorial rotation velocity, instead of assuming spherical symmetry as was done in Paper I. We first outline the basic assumptions we made for our calculations before providing the final results.

\subsection{Critical rotation velocity}

The equatorial velocity $v_{\mathrm{eq}}$ is varied from slow rotation up to critical rotation velocity, which is given by $v_{\text {crit }}=\sqrt{2 G M / 3 R_{\text {polar }}}$ whenever the Eddington factor $\Gamma<0.64$ (Maeder \& Meynet 2000). For $\Gamma>0.64$, however, Maeder \& Meynet (2000) have shown the critical velocity to decrease significantly because of the reduced effective escape velocity, and in that case we have adopted values for $v_{\text {crit }}$ according to their Fig. 1. We will express the equatorial rotation velocity in terms of $v_{\text {crit }}$ in the rest of this paper.

\subsection{The shape of the star}

The surface of an oblate rotating star has an inhomogeneous temperature and flux distribution. We assume the Von Zeipel effect to be valid, which is a good approximation for radiative stellar envelopes. The Von Zeipel theorem expresses that the effective radiative flux from a point on a distorted star is proportional to the local effective gravity, which can be determined by taking into account the real shape of a uniformly rotating star. In doing so, we assume that all the stellar mass is concentrated in the stellar centre. In that case, the shape of the star is determined by its Roche equipotential surfaces, which take the following dimensionless form in a co-rotating frame of reference:

$\Phi(x, y, \theta)=\frac{1}{x}+\frac{1}{2} x^{2} y^{2} \sin ^{2} \theta$,

where $x \equiv r(\theta, y) / R_{\mathrm{eq}}$, and $y=v_{\mathrm{eq}} / v_{\text {crit }}$ (see, e.g. Maeder $\&$ Meynet 2000, Eq. (A.6), for the equivalent formulation in terms of $\left.x=r(\theta, y) / R_{\text {pole }}\right)$. The local radius $x$ of the star in such a Roche model is given by

$x(\theta, y)=2 \frac{\sqrt{\left(2+y^{2}\right)}}{\sqrt{3} y \sin \theta} \sin \left[\frac{1}{3} \arcsin \left(\frac{3 \sqrt{3} y \sin \theta}{\left(2+y^{2}\right)^{3 / 2}}\right)\right]$

(see Eq. (4) in Pelupessy et al. 2000) and is graphically depicted in the upper left panel of Fig. 1.

Through the Von Zeipel theorem we are able to calculate the latitudinal dependence of the radiative flux for a particular $y$ :

$|\boldsymbol{g}(\theta, y)|=|\boldsymbol{\nabla} \Phi(\theta, y)| \sim T_{\mathrm{eff}}^{4}(\theta, y)$

from the potential $\Phi$. A plot of the latitudinal dependence of the relative gravity, i.e. of the ratio of the local gravity to the gravity at the equator, and from this of the relative local effective temperature, is provided in Fig. 1. 

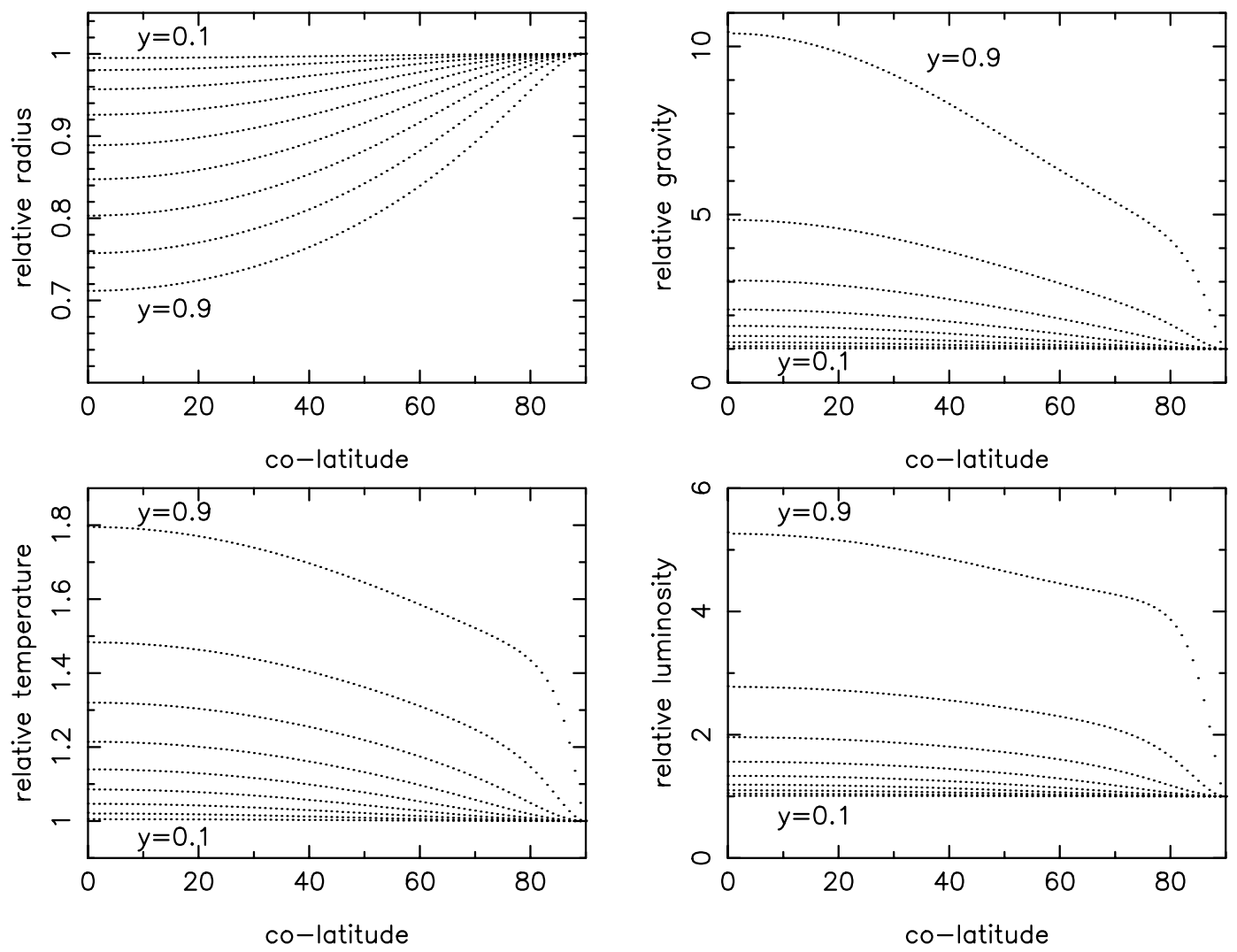

Fig. 1. The local radius (upper left), local gravity (upper right), local effective temperature (lower left) and local luminosity $L(\theta) \equiv$ $4 \pi R^{2}(\theta) T_{\mathrm{eff}}^{4}(\theta)$ (lower right) relative to the equatorial value of these quantities, for different ratios of the equatorial velocity to the critical velocity and as a function of co-latitude $\left(\theta=0\right.$ at the pole). The step in $y=v_{\text {eq }} / v_{\text {crit }}$ for the different curves is 0.1 .

By multiplication of $x^{2}$ and $T_{\text {eff }}^{4}$, we also obtain the relative local luminosity as a function of co-latitude (see Fig. 1, lower right panel). In Fig. 1, the oblateness of the star is expressed in terms of $y$, which is increased from $y=0$ (spherical star) to $y=0.9$ in steps of 0.1 for the different dotted curves in the panels.

\subsection{A sectorial model}

In our model calculations we ignore any $\theta$-component of the flow velocity and we solve the momentum equation in one dimension (viz. the radial direction) only, i.e. we solve the momentum equation (Eq. (9) of Paper I) for the values of the relevant parameters for each co-latitude $\theta$. We thus neglect any wind compression due to velocity components towards the equatorial plane that may occur, as described by Bjorkman \& Cassinelli (1993). This assumption is justified because compression merely redistributes the wind material and does not change the total mass lost from the star, which interests us here. In this approximation, we write the conservation of mass loss for a non-spherical sectorial wind as

$$
\begin{aligned}
\dot{M}(\theta, y) & \equiv 4 \pi R^{2}(\theta, y) F_{m}(\theta, y) \\
& =4 \pi r^{2}(\theta, y) \rho[r(\theta, y)] v[r(\theta, y)],
\end{aligned}
$$

with $\dot{M}(\theta, y)$ the local mass-loss rate and $F_{m}(\theta, y)$ the local mass flux along the surface normal for a particular equatorial rotation velocity $y=v_{\text {eq }} / v_{\text {crit }}$ (see Fig. 2).

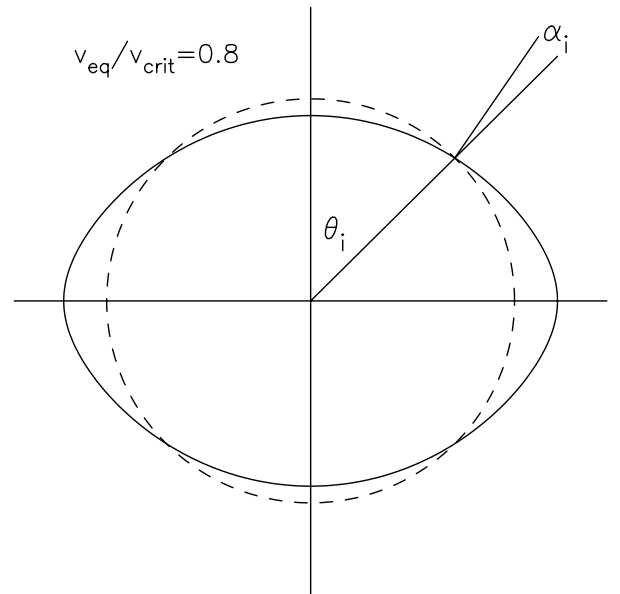

Fig. 2. A rotationally distorted star according to the Roche model for $v_{\text {eq }} / v_{\text {crit }}=0.8$ (full line). At a certain co-latitude $\theta_{i}$ the surface normal to the distorted star encloses an angle $\alpha_{i}$ with the radial vector, which is perpendicular to a spherical star with radius $r\left(\theta_{i}\right)$ (dashed line). The surface-integrated maximum mass loss calculated from Exp. (5) takes into account the maximum mass flux along the normal to the true stellar surface.

At different values of $\theta$, the equation to solve for the maximum mass-loss rate at that $\theta$ differs because the local luminosity, the local radius and the local effective temperature change for each co-latitude $\theta$ (according to Fig. 1). Moreover, also the Eddington factor depends on the co-latitude and on the 
rotation (see Maeder \& Meynet 2000). For uniform rotation the $\theta$-dependence of the Eddington factor solely occurs in the opacity. We assume that the only source of continuum opacity in the envelope is electron scattering, which is a good approximation for the high surface temperatures considered here. We have taken the continuum opacity values from Lamers \& Leitherer (1993) - see Table 1. Hence, the opacity, and therefore also the Eddington factor, is independent of the co-latitude. We are then only left with a rotational dependence of $\Gamma$, according to Eq. (4.28) in Maeder \& Meynet (2000), which we adopt in this work.

The change of the finite disk correction factor due to stellar oblateness and to the $\theta$-dependence of $T_{\text {eff }}$ is neglected. As shown in Paper I, the inclusion of the finite disk correction factor has only a small influence of a few percent on maximum mass-loss rates determined from our formalism (contrary to its large effect for the classical CAK solution). It is therefore justified to keep its value for a spherical star for all co-latitudes.

\subsection{The force multipliers}

The values of the force multipliers $k$ and $\alpha$ are listed in Table 1 and were taken from Pauldrach et al. (1986) for the different temperature ranges. The force multiplier parameter $\delta$ was set to zero for reasons outlined in Paper I. For the calculation of the mass loss we take into account the change in the value of $k$ and $\alpha$ across the stellar surface due to the varying temperature, according to Fig. 1.

\subsection{Strategy}

We determined the local maximum mass flux through a sphere with radius $r(\theta, y)$ (see dashed line in Fig. 2) at a particular co-latitude $\theta$ and for a particular value of the equatorial rotation velocity $y$ by numerical integration of the momentum equation. We did this by using the appropriate values of the local radius, local effective temperature (and corresponding $\alpha, k$ and $\sigma_{\mathrm{e}}$-values), local luminosity and local Eddington factor in Eq. (9) of Paper I. This procedure was followed for 4 different values of the co-latitude $\theta: 90^{\circ}, 60^{\circ}, 30^{\circ}$ and $0^{\circ}$; for 10 different values of the ratio $y=v_{\text {eq }} / v_{\text {crit }}$ ranging from 0 to 1 in steps of 0.1 and for the 9 stellar models of which the equatorial parameters are given in Table 1 . This leads to 360 local maximum mass-loss determinations $\dot{M}_{\max }^{\text {sphere }}(\theta, y)$.

As already mentioned, the mass flux does not occur along the radial direction but rather perpendicular to the local surface (see Fig. 2). When calculating the surface-integrated maximum mass loss we corrected for this difference in flux direction by projecting the local radial vector onto the local surface normal. We thus approximate the surface-integrated mass-loss by

$\dot{M}_{\max }^{\mathrm{tot}}(y)=\frac{1}{4} \sum_{i=1}^{4} \dot{M}_{\max }^{\mathrm{sphere}}\left(\theta_{i}, y\right) \cos \alpha_{i}$

where $\theta_{i}=0^{\circ}, 30^{\circ}, 60^{\circ}, 90^{\circ}$ while $\alpha_{i}$ is the angle between the local surface normal at co-latitude $\theta_{i}$ for $i=1,2,3,4$ and the local radial vector (see Fig. 2 for an example with $y=0.8$ and $\left.\theta_{i}=45^{\circ}\right)$. With this definition of $\dot{M}_{\mathrm{max}}^{\mathrm{tot}}(\theta)$ the total loss of angular momentum is approximated by:

$-\dot{J}_{\max }^{\mathrm{tot}}(y)=\frac{1}{4} \Omega R_{\mathrm{eq}}^{2} \sum_{i=1}^{4} \dot{M}_{\max }^{\text {sphere }}\left(\theta_{i}, y\right) \cos ^{3} \alpha_{i} x^{2}\left(\theta_{i}, y\right)$,

with $\Omega \equiv v_{\text {eq }} / R_{\text {eq }}$.

\subsection{Results}

We provide in Table 2 the equatorial, polar and surfaceintegrated maximum mass-loss rates with the corresponding maximum loss of angular momentum of the 9 models with equatorial stellar parameters given in Table 1, as a function of $y$. We find that the maximum mass-loss rates induce a bipolar prolate form, in agreement with results of earlier studies (e.g. Dwarkadas \& Owocki 2002). This form becomes more outspoken as $y$ increases. We also find that the inclusion of the Von Zeipel effect and the oblateness of the star has a significant effect on the ratio of the maximum mass-loss rate for a rotating star to that one of the non-rotating star. The factor 2-3 increase in maximum mass loss from no rotation to critical rotation found in Paper I is largely exceeded in the present work.

The onset of extreme mass loss is different for the different stellar models, occurring already at $y=0.5$ for stars with a high Eddington factor (above 0.6 on the entire surface, e.g. Model 3 ) and only at $y=0.9$ for stars with a very low Eddington factor (below 0.2 everywhere on the surface such as for Models $7,8,9)$. The allowance of a possible extra force at the base of the stellar wind thus implies that extreme mass loss might occur at critical rotation, for any stellar model. This is in contrast to the situation where extra forces are not taken into account and the mass loss is finite if the star is sufficiently far from the $\Omega \Gamma$-limit (Owocki et al. 1998; Maeder \& Meynet 2000). On the other hand, to reach the stage of extreme mass loss, one needs a rotation rate that is at least about half the critical rate for the models we considered here. This threshold decreases considerably for a star closer to the Eddington limit, as we show in the next section and as was already found by Maeder \& Meynet (2000). If the extreme mass loss occurs at any lattitude, the star can no longer be considered to be stable.

In Fig. 3 we plot the ratio of $\dot{M}_{\text {max }}^{\text {tot }}\left(v_{\mathrm{eq}}\right)$ and $\dot{M}_{\text {max }}^{\text {tot }}\left(v_{\mathrm{eq}}=0\right)$ versus $v_{\text {eq }} / v_{\text {crit }}$ as filled squares for the 9 models. The maximum mass-loss rate increases drastically whenever the star rotates faster than half the critical velocity. Globally, the curves behave rather similar for all 9 models and point towards a moderate increase in $\dot{M}_{\text {max }}^{\text {tot }}\left(v_{\text {eq }}\right)$ as a function of the rotation velocity for $v_{\text {eq }} / v_{\text {crit }}<0.4$ and a very steep increase for faster rotation. As expected, for models of the same temperature, the onset of extreme mass loss occurs at higher rotation as the luminosity decreases (compare e.g. Models 2, 4, 7).

\subsubsection{Comparison with previous estimates}

The dotted line in the panels of Fig. 3 is the empirically derived relation proposed by Langer (1998) for self-regulated winds (i.e. without the inclusion of a possible extra force at the base of the wind that might increase the mass loss rate), applied 
Table 2. Surface-integrated maximum mass-loss rates (expressed in $10^{-6} M_{\odot} \mathrm{yr}^{-1}$ ) and corresponding maximum angular momentum loss (expressed in $10^{37} \mathrm{~kg} \mathrm{~m}^{2} \mathrm{~s}^{-2}=1.94 \times 10^{-3} \mathrm{~J}_{\odot} \mathrm{yr}^{-1}$ ) for the stellar models of which the equatorial parameters are listed in Table 1, as a function of the rotation velocity. The indication " $\infty$ " means that the star has reached the $\Omega \Gamma$-limit.

\begin{tabular}{|c|c|c|c|c|c|c|c|c|c|c|c|c|}
\hline$v_{\text {eq }} / v_{\text {crit }}$ & $\dot{M}_{\max }^{\mathrm{eq}}$ & $\dot{M}_{\max }^{\text {pole }}$ & $\dot{M}_{\max }^{\text {tot }}$ & $-\dot{J}_{\max }^{\mathrm{tot}}$ & $\dot{M}_{\max }^{\mathrm{eq}}$ & $\dot{M}_{\max }^{\text {pole }}$ & $\dot{M}_{\max }^{\text {tot }}$ & $-\dot{J}_{\max }^{\mathrm{tot}}$ & $\dot{M}_{\max }^{\mathrm{eq}}$ & $\dot{M}_{\max }^{\text {pole }}$ & $\dot{M}_{\text {max }}^{\text {tot }}$ & $-j_{\text {max }}^{\text {tot }}$ \\
\hline & \multicolumn{4}{|c|}{ Model 1} & \multicolumn{4}{|c|}{ Model 4} & \multicolumn{4}{|c|}{ Model 7} \\
\hline 0.2 & 4.7 & 5.1 & 4.9 & 2.27 & 0.9 & 0.9 & 0.9 & 1.09 & 0.1 & 0.2 & 0.2 & 0.14 \\
\hline 0.3 & 5.3 & 6.3 & 5.8 & 3.95 & 0.9 & 1.1 & 1.0 & 1.85 & 0.2 & 0.2 & 0.2 & 0.23 \\
\hline 0.4 & 6.2 & 9.3 & 7.7 & 6.73 & 1.0 & 1.4 & 1.2 & 2.97 & 0.2 & 0.2 & 0.2 & 0.37 \\
\hline 0.5 & 8.2 & 18.3 & 13.7 & 13.89 & 1.2 & 1.9 & 1.6 & 4.89 & 0.2 & 0.3 & 0.3 & 0.60 \\
\hline 0.6 & 13.1 & $\infty$ & $\infty$ & $\infty$ & 1.6 & 3.3 & 2.7 & 9.69 & 0.3 & 0.5 & 0.4 & 1.06 \\
\hline 0.7 & $\infty$ & $\infty$ & $\infty$ & $\infty$ & 2.4 & 8.4 & 6.1 & 25.55 & 0.3 & 0.9 & 0.7 & 2.13 \\
\hline 0.8 & $\infty$ & $\infty$ & $\infty$ & $\infty$ & 3.1 & $\infty$ & $\infty$ & $\infty$ & 0.4 & 1.9 & 1.4 & 5.06 \\
\hline \multirow[t]{2}{*}{0.9} & $\infty$ & $\infty$ & $\infty$ & $\infty$ & $\infty$ & $\infty$ & $\infty$ & $\infty$ & 0.6 & $\infty$ & $\infty$ & $\infty$ \\
\hline & \multicolumn{4}{|c|}{ Model 2} & \multicolumn{4}{|c|}{ Model 5} & \multicolumn{4}{|c|}{ Model 8} \\
\hline 0.2 & 6.7 & 7.4 & 7.0 & 2.40 & 1.1 & 1.2 & 1.1 & 0.21 & 0.2 & 0.2 & 0.2 & 0.02 \\
\hline 0.3 & 7.7 & 9.5 & 8.6 & 4.44 & 1.2 & 1.4 & 1.3 & 0.37 & 0.2 & 0.2 & 0.2 & 0.03 \\
\hline 0.4 & 9.4 & 16.3 & 12.8 & 8.79 & 1.4 & 1.9 & 1.6 & 0.62 & 0.2 & 0.3 & 0.2 & 0.05 \\
\hline 0.5 & 14.1 & $\infty$ & $\infty$ & $\infty$ & 1.7 & 2.8 & 2.3 & 1.08 & 0.2 & 0.4 & 0.3 & 0.08 \\
\hline 0.6 & 19.0 & $\infty$ & $\infty$ & $\infty$ & 2.3 & 5.5 & 4.2 & 2.36 & 0.3 & 0.6 & 0.5 & 0.15 \\
\hline 0.7 & $\infty$ & $\infty$ & $\infty$ & $\infty$ & 3.3 & 13.3 & 9.2 & 5.98 & 0.5 & 1.3 & 0.9 & 0.33 \\
\hline 0.8 & $\infty$ & $\infty$ & $\infty$ & $\infty$ & 4.1 & $\infty$ & $\infty$ & $\infty$ & 0.6 & 3.5 & 2.3 & 0.90 \\
\hline \multirow[t]{2}{*}{0.9} & $\infty$ & $\infty$ & $\infty$ & $\infty$ & $\infty$ & $\infty$ & $\infty$ & $\infty$ & 0.7 & $\infty$ & $\infty$ & $\infty$ \\
\hline & \multicolumn{4}{|c|}{ Model 3} & \multicolumn{4}{|c|}{ Model 6} & \multicolumn{4}{|c|}{ Model 9} \\
\hline 0.2 & 13.6 & 15.7 & 14.4 & 4.90 & 3.7 & 4.0 & 3.8 & 0.75 & 0.6 & 0.6 & 0.6 & 0.06 \\
\hline 0.3 & 16.8 & 23.6 & 20.0 & 10.21 & 4.1 & 4.9 & 4.4 & 1.31 & 0.6 & 0.7 & 0.6 & 0.09 \\
\hline 0.4 & 20.1 & 52.9 & 34.6 & 19.87 & 4.8 & 6.8 & 5.7 & 2.26 & 0.7 & 0.9 & 0.8 & 0.15 \\
\hline 0.5 & 32.2 & $\infty$ & $\infty$ & $\infty$ & 6.1 & 10.5 & 8.5 & 4.17 & 0.8 & 1.3 & 1.0 & 0.25 \\
\hline 0.6 & 40.4 & $\infty$ & $\infty$ & $\infty$ & 8.8 & 24.3 & 17.7 & 10.2 & 1.1 & 2.3 & 1.7 & 0.48 \\
\hline 0.7 & $\infty$ & $\infty$ & $\infty$ & $\infty$ & 11.9 & $\infty$ & $\infty$ & $\infty$ & 1.7 & 5.6 & 3.7 & 1.16 \\
\hline 0.8 & $\infty$ & $\infty$ & $\infty$ & $\infty$ & $\infty$ & $\infty$ & $\infty$ & $\infty$ & 2.1 & 14.0 & 8.7 & 2.95 \\
\hline 0.9 & $\infty$ & $\infty$ & $\infty$ & $\infty$ & $\infty$ & $\infty$ & $\infty$ & $\infty$ & $\infty$ & $\infty$ & $\infty$ & $\infty$ \\
\hline
\end{tabular}

to our maximum equatorial mass-loss rates for the 9 stellar models listed in Table 1. Langer (1998) neglected the oblateness of the star and the Von Zeipel effect. His relation leads to mass-loss rates that are overestimates of the true values if one does not include extra forces near the sonic point. This can be seen by comparison with the correct scaling law derived by Maeder \& Meynet (2000) for a self-regulated CAK-type wind (dashed line in Fig. 3). If one allows instabilities, though, one easily ends up with larger mass-loss rates than those predicted by Langer's relation.

We find systematically a significantly higher contrast in the maximum mass-loss rate with and without rotation than the one found by Maeder \& Meynet (2000). This finding shows that the inclusion of extra forces at the base of the wind allows the onset of extreme mass loss at much lower rotation rates compared to the situation where such extra forces do not occur. It is entirely understandable that we find a larger contrast than Maeder \& Meynet (2000) who did not include any additional helpful force. Maeder \& Meynet (2000) had already derived extreme mass loss from their scaling law for stars close to the so-called $\Omega \Gamma$-limit. We confirm this result by direct numerical integration of the momentum equation and we show that the onset of extreme mass loss occurs at lower rotation rates whenever extra forces help to lift the material at the stellar surface.

Langer (1998) computed the main sequence evolution of a $60 M_{\odot}$ star with various initial rotation rates and considered the effect of angular momentum loss on the stellar mass-loss rate and the rotation of the star. He finds that the coupling of mass and angular momentum loss limits the mass-loss rate of main-sequence stars at the so-called $\Omega$-limit. He lists a value of $\dot{M}_{\max }^{\text {tot }} \simeq 10^{-5} M_{\odot} \mathrm{yr}^{-1}$ which is determined through the angular momentum loss imposed by the $\Omega$-limit. We have done quite opposite calculations as we have built up a formalism to find the maximum mass-loss rate by line-driving from a physical model and we derived the accompanied maximum angular momentum loss. The maximum mass loss of the slowly rotating O-star models in our sample is indeed of the order $10^{-5} M_{\odot} \mathrm{yr}^{-1}$, which is compatible with Langer's (1998) result. Hence, there seems to be general agreement between our results and stellar evolution calculations of massive stars.

\subsubsection{The effect of the force multiplier parameters}

The values of the maximum mass-loss rates listed in Table 2, and hence also those of the maximum angular momentum loss, are determined by the adopted values of the line force parameters $(\alpha, k)$. It is clear that any future change in the values of these parameters will imply different values for the maximum mass-loss rates. This should always be kept in mind when using them for stellar evolution calculations. The main purpose of our work was to investigate how high a mass can be driven in the best possible circumstances of having a high rotation and 


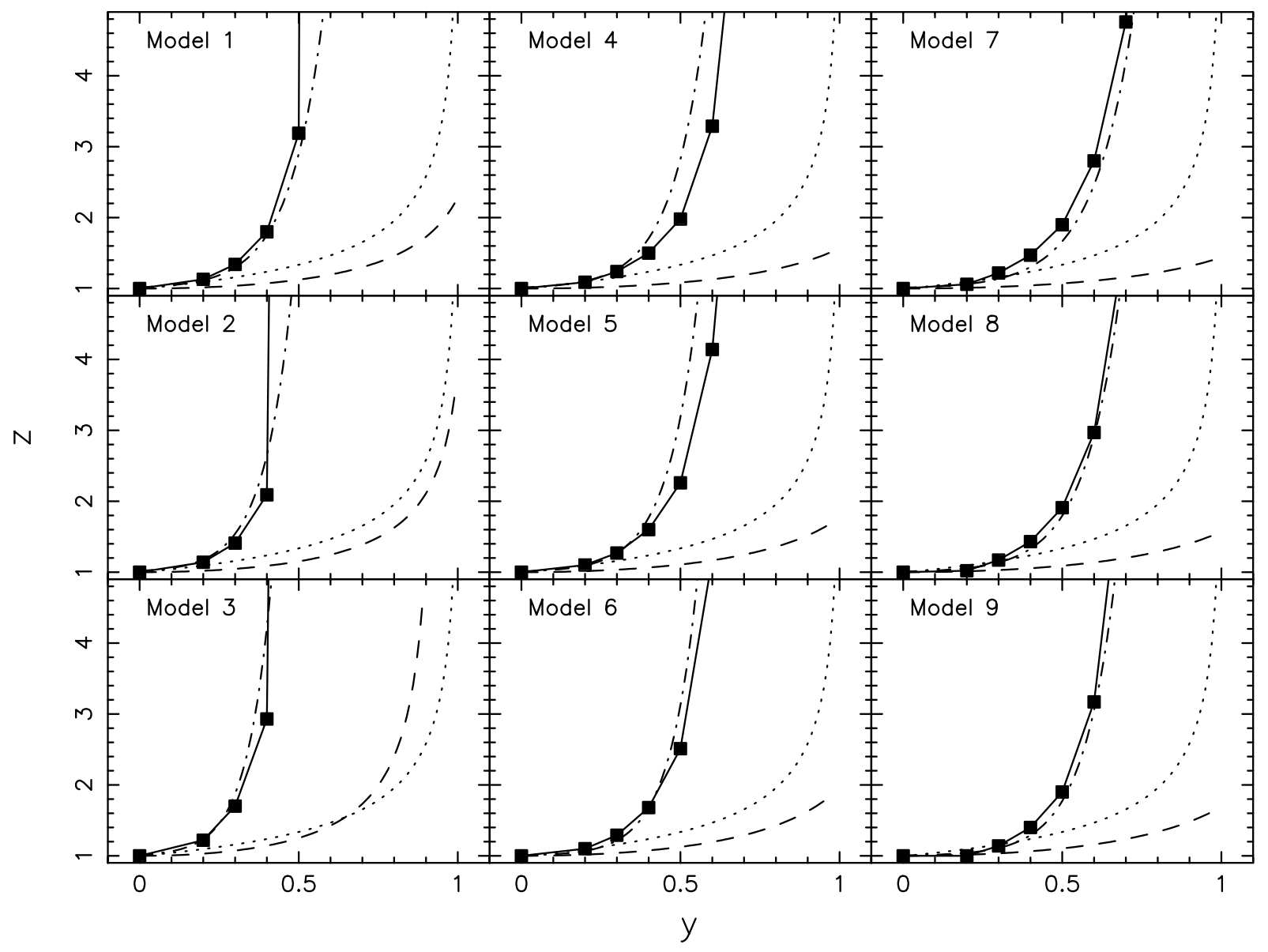

Fig. 3. $z \equiv \dot{M}_{\max }^{\text {tot }}\left(v_{\text {eq }} / v_{\text {crit }}\right) / \dot{M}_{\max }^{\text {tot }}(0)$ as a function of $y \equiv v_{\mathrm{eq}} / v_{\text {crit }}$ for rotating models including the Von Zeipel effect. The values are obtained from direct integration of the momentum equation at different co-latitudes and are indicated by filled boxes connected by a full line. The dotted line represents the empirical relation proposed by Langer (1998). The scaling law proposed by Maeder \& Meynet (2000) for winds in rotating stars without instabilities is superimposed as a dashed line. The dashed-dotted line represents the multiple regression prediction formula given in Exps. (8) and (9).

extra forces helping to lift the wind material, compared to the case where rotation does not occur. This ratio should not be very sensitive to the line-force parameters. We have taken the values by Pauldrach et al. (1986) derived from theory rather than empirical values derived from observations. The reason why we did not rely on the empirical values is that we needed a consistent set of $(\alpha, k)$ at each temperature at the stellar surface to be able to solve the momentum equation rather than using a scaling law to predict the mass loss. We also note for completeness that the empirical $\alpha_{m}$-values listed by Lamers et al. (1995) are too low due to a small error in the code that was used by the authors to derive them.

\section{Maximum mass-loss recipe}

The observation that the dependence of the increase of the maximum mass-loss rates on the ratio $v_{\text {eq }} / v_{\text {crit }}$ is quite similar over a wide range of stellar models, as illustrated in Fig. 3, is very fortunate. Indeed, it permits us to derive a formula that allows the prediction of the maximum mass-loss rates for a star with a particular luminosity, effective temperature and mass, as a function of the equatorial rotation velocity, instead of having to calculate each time again the $\dot{M}_{\max }^{\text {tot }}$-values when the stellar model parameters deviate from those given in Table 1. With the specific goal of determining such a statistical formulation we have performed multiple regression, using the procedure NLMIXED of the statistical software package SAS (2002).

We have considered several non-linear model options and the best result was obtained for a logistic function, which finally leads to the form

$z_{i}=\frac{1+\exp \left(f\left(y_{i}, L, M, T_{\mathrm{eff}}\right)\right)}{\exp \left(f\left(y_{i}, L, M, T_{\mathrm{eff}}\right)\right)}+\varepsilon_{i}$

with $z_{i} \equiv \dot{M}_{\max }^{\text {tot }}\left(y_{i}\right) / \dot{M}_{\max }^{\text {tot }}(0)$ and $y_{i} \equiv v_{\text {eq }} / v_{\text {crit }}$ for each of $i=1, \ldots, 81$. In this model we assume normally distributed errors $\varepsilon_{i} \sim \mathcal{N}\left(0, \sigma^{2}\right)$ and the function $f\left(y, L, M, T_{\text {eff }}\right)$ is taken to be linear in $y$ and dependent of the luminosity, mass and temperature of the star. In the first instance, we determined the best fit for each of the 9 stellar models separately. This leads to very satisfactory fits, which is not surprising as we have a large degree of freedom with only 9 points to fit.

A subsequent important question is: can we find one global non-linear model, with a good predictive power, that describes the data of the 9 models simultaneously? If so, we can predict $\dot{M}_{\max }^{\text {tot }}\left(v_{\text {eq }}\right)$ from the maximum mass-loss rate at zero rotation as a function of mass, luminosity, effective temperature and 
equatorial rotation speed for the range of physical parameters listed in Table 1 . The coefficients for the linear function $f$ were hence determined from one global fit to the 81 calculated values of $\dot{M}_{\max }^{\text {tot }}$ (shown as full squares in Fig. 3) simultaneously. We started from the most general linear function for $f$ and kept only those terms with significant coefficients. This results in the following maximum mass-loss recipe:

$$
\dot{M}_{\text {max }}^{\text {tot }}\left(v_{\text {eq }}\right)=\dot{M}_{\text {max }}^{\text {tot }}\left(v_{\mathrm{eq}}=0\right) \times \frac{1+\exp (f(y))}{\exp (f(y))}
$$

with

$$
\begin{aligned}
& f\left(y, L, M, T_{\text {eff }}\right)=27.72-1.19 \log \left(L / L_{\odot}\right)+0.04 M \\
& -4.05 \log T_{\text {eff }}+22.02 y-7.12 \log \left(L / L_{\odot}\right) y+0.19 M y,
\end{aligned}
$$

where $y=v_{\text {eq }} / v_{\text {crit }}$ and $v_{\text {crit }}=\sqrt{2 G M / 3 R_{\text {polar }}}$ for $\Gamma<0.64$ and as given in Maeder \& Meynet (2000) for higher values of $\Gamma$. The global fits for the 9 models based on Eqs. (8) and (9) are shown as dashed-dot lines in Fig. 3. It can be seen that the fits are satisfactory from a point of view of providing accurate maximum mass-loss estimates as input to stellar evolution calculations. The fit allows one to predict the maximum mass loss as a function of equatorial rotation velocity, provided that the overall maximum mass loss without rotation is known, for all stellar parameters not too different from those in our grid of 9 models.

As shown in Paper I (see last column in Table 3 of that paper), the maximum mass-loss rate of a non-rotating star is between 0.5 and 1.7 times the rate of a line-driven wind calculated with the inclusion of multiple scattering for solar metallicity (see Vink et al. 2000). It is therefore a very good approximation to use the values from the recipe provided by Vink et al. (2000) for solar metallicity, in combination with our scaling law (8) and (9) in order to find the maximum mass than can be lost by a star as a function of rotation velocity. For completeness, we therefore repeat here the results obtained by Vink et al. (2000):

$$
\begin{aligned}
& \log \dot{M}=-6.70+2.19 \log \left(L / 10^{5}\right)-1.31 \log (M / 30) \\
& -1.23 \log \left(v_{\infty} / 2 v_{\text {esc }}\right)+0.93 \log \left(T_{\text {eff }} / 40000\right) \\
& -10.92\left\{\log \left(T_{\text {eff }} / 40000\right)\right\}^{2}
\end{aligned}
$$

in the range of $25000<T_{\text {eff }}<50000 \mathrm{~K}$ where $v_{\infty}=2.6 v_{\text {esc }}$, and

$$
\begin{gathered}
\log \dot{M}=-6.69+2.21 \log \left(L / 10^{5}\right)-1.34 \log (M / 30) \\
-1.60 \log \left(v_{\infty} / 2 v_{\text {esc }}\right)+1.07 \log \left(T_{\text {eff }} / 20000\right)
\end{gathered}
$$

in the range of $15000<T_{\text {eff }}<22500 \mathrm{~K}$, where $v_{\infty}=1.3 v_{\text {esc }}$. The parameters $L$ and $M$ have to be expressed in solar units in these formulae. These multiple scattering mass-loss predictions can be rescaled to include the effect of deviations from solar metallicity according to Vink et al. (2001).

\section{The case of $\eta$ Carinae and other luminous blue variables}

As already emphasized by Maeder \& Meynet (2000), extreme mass loss occurs for stars near the $\Omega \Gamma$-limit, such as luminous blue variables (LBVs). Having included possible extra forces or instabilities to help build up the ideal velocity gradient at the sonic point, we can check if our models are able to explain the mass loss and the kinematical structure of nebulae around such stars, which are indeed expected to undergo instabilities. Weiss (2003) recently provided an overview of such structures for several galactic and LMC LBVs. Given its extreme properties, we concentrate mainly on the LBV $\eta$ Carinae and we confront its observational characteristics with our maximum mass-loss formalism.

Maeder \& Desjacques (2001) used their mass-loss scaling law to derive mass flux plots of stars like $\eta$ Carinae in the two cases of a shell ejection and of a constant wind. A much more systematic study of the shaping of LBV nebulae in terms of radiatively driven winds was provided by Dwarkadas $\&$ Owocki (2002). They obtained an equatorial mass-loss rate that is about $1 / 5$ of its polar value. They also provided density contours derived from wind simulations for which they fixed the maximum mass-loss rate to $10^{-4} M_{\odot} \mathrm{yr}^{-1}$ and the maximum polar wind velocity to $2000 \mathrm{~km} \mathrm{~s}^{-1}$. Their simulations showed convincingly that radiatively driven wind theory leads in a natural way to a latitudinal dependence in velocity and mass loss for rotating stars, with both a higher mass flux and a higher terminal velocity from the pole than from the equator (in the absence of a bi-stability jump). They also provided a model for $\eta$ Carinae in which they assumed a pre-outburst wind with a polar velocity of $700 \mathrm{~km} \mathrm{~s}^{-1}$ and a mass loss of $10^{-5} M_{\odot} \mathrm{yr}^{-1}$, and an outburst period of 20 years during which the velocity was assumed not to change but during which the mass loss increased to $0.1 M_{\odot} \mathrm{yr}^{-1}$. The density contour plots they obtained in this way indeed resemble $\eta$ Carinae's Homunculus Nebula.

Recent progress in determining the observational properties of $\eta$ Carinae is impressive. Hillier et al. (2001) have derived observational properties of the star from HST/STIS spectra and require a current mass-loss rate of $10^{-3} M_{\odot} \mathrm{yr}^{-1}$ to obtain a good fit to the spectrum. More recently, van Boekel et al. (2003) have obtained a direct measurement of the size and the shape of the stellar wind of $\eta$ Carinae from interferometric data gathered with the VLTI. They derive an even higher current massloss rate of $1.6 \pm 0.3 \times 10^{-3} M_{\odot} \mathrm{yr}^{-1}$, assuming a spherically symmetric clumped wind. Smith et al. (2003) found the wind structure of the star to be axisymmetric and variable in time, with higher velocities (of order $600-1000 \mathrm{~km} \mathrm{~s}^{-1}$ ) and higher densities at the pole than at the equator from new STIS spectra.

An important question is whether the high observed massloss rate of $\eta$ Carinae can be explained in terms of radiatively driven wind theory. Our formalism is ideally suited to answer this question as it allows for the influence of instabilities at the base of the wind. We have therefore solved the momentum equation for stellar parameters appropriate for $\eta$ Carinae and determined the maximum mass-loss rates as explained in Sect. 3 for models that have not yet reached the $\Omega \Gamma$-limit but are only barely below this limit. We have kept a large degree of freedom on stellar parameters in the first instance as these are not well known for $\eta$ Carinae.

It is not our intention to provide a fully explored set of models that lead to the observed mass loss. Rather we emphasize that such models indeed were found by us. One such 


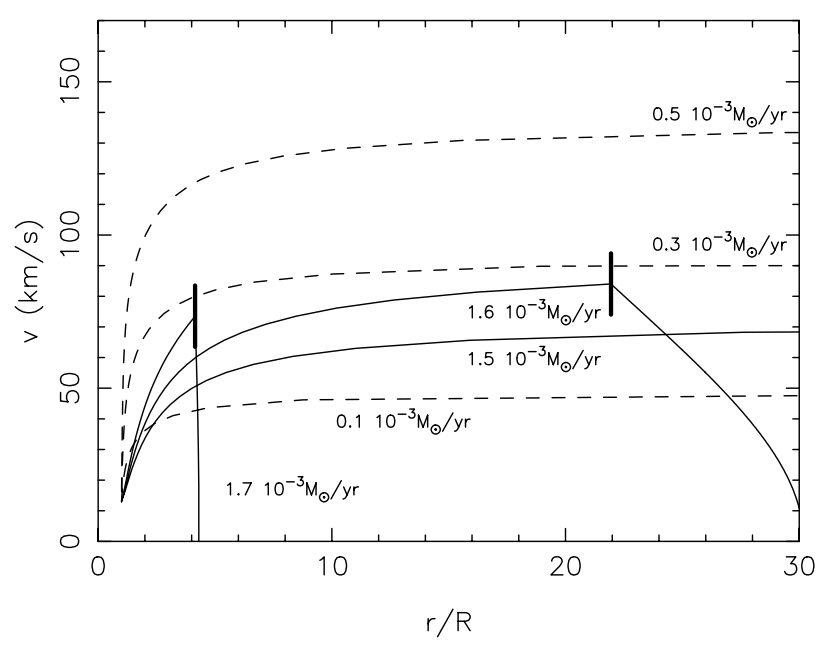

Fig. 4. Some velocity laws resulting from integration of the momentum equation for stellar parameters appropriate for $\eta$ Car, allowing for different values of the velocity gradient at the sonic point. The full lines are solutions for the polar region while the dashed lines are valid for the equatorial regions. The mass-loss rates corresponding to the velocity laws are indicated. For a more detailed explanation we refer to Paper I and to the text.

model has the following characteristics: $M=90 M_{\odot}, R_{\mathrm{eq}}=$ $60 R_{\odot}, R_{\text {pole }}=57.4 R_{\odot}, T_{\mathrm{eff}}^{\mathrm{eq}}=20000 \mathrm{~K}, T_{\mathrm{eff}}^{\text {pole }}=20935 \mathrm{~K}$, $\log \left(L / L_{\odot}\right)^{\mathrm{eq}}=6.50, \log \left(L / L_{\odot}\right)^{\text {pole }}=6.54, v_{\text {crit }}=250 \mathrm{~km} \mathrm{~s}^{-1}$, $v_{\text {eq }} / v_{\text {crit }}=0.3, \Gamma=0.87$. The highest possible mass-loss rates we obtained from integration of the momentum equation are in this case $1.5 \times 10^{-3} M_{\odot} \mathrm{yr}^{-1}$ at the pole and $0.5 \times 10^{-3} M_{\odot} \mathrm{yr}^{-1}$ at the equator, leading to a surface-integrated mass loss of $10^{-3} M_{\odot} \mathrm{yr}^{-1}$. The mass-loss contrast of a factor three between the pole and the equator (with a higher mass loss rate from the pole), together with the factor 0.5 for the maximum velocity, (with a higher outflow velocity from the equator - see below) leads to a density contrast of a factor 6 . This is compatible with the factor 5 found by Dwarkadas \& Owocki (2002), although they obtain a higher velocity at the pole than at the equator. We stress that the listed model parameters are just one set that lead to the appropriate mass-loss rate and there may be several others that do so as well. We also stress that much more extreme mass-loss rates are easily reached for stars above the $\Omega \Gamma$-limit (i.e. with $v_{\text {eq }} / v_{\text {crit }}>0.4$ ), as shown in Fig. 3 and listed in Table 2 in Sect. 3. However, the purpose of our investigation of $\eta$ Carinae was to search for models with a "natural" linedriven outflow based on an ideal velocity gradient at the sonic point that explains the observed mass loss rather than having to invoke a specific outburst with a much increased ad-hoc massloss rate as often assumed in the literature. From the point of view of the high mass loss we have succeeded in finding such models.

The velocity laws accompanying the solution for the model mentioned above are shown in Fig. 4. The full lines are for the polar region of the star. It can be seen that a kinked velocity law (see Paper I) is found as of $\dot{M}_{\max }^{\text {pole }}=1.6 \times 10^{-3} M_{\odot} \mathrm{yr}^{-1}$ for the polar mass loss and the matter can no longer escape from the star if the mass-loss rate is further increased to $1.7 \times 10^{-3} M_{\odot} \mathrm{yr}^{-1}$. We refer to Paper I for a more detailed description of the velocity laws in this figure. The dashed lines indicate the three acceptable solutions with the highest possible mass loss for the equatorial region. Increasing the mass loss above $\dot{M}_{\max }^{\mathrm{eq}}=0.5 \times 10^{-3} M_{\odot} \mathrm{yr}^{-1}$ implies fall-back of the wind material in the equatorial zone. The maximum wind velocity near the pole for the model shown in Fig. 4 is only some $80 \mathrm{~km} \mathrm{~s}^{-1}$ and cannot explain the extent of the Homunculus if it formed only after the outburst around 1850.

While large uncertainties of several hundred $\mathrm{km} \mathrm{s}^{-1}$ exist for the terminal wind velocity of $\eta$ Carinae we could not find a model below the $\Omega \Gamma$-limit that explains both the observed mass-loss rate and the wind velocity of the star in full details. For appropriate mass-loss rates, we always end up with a much lower wind velocity, similar to the one shown in Fig. 4. Note that Maeder \& Desjaques (2001) and Dwarkadas \& Owocki (2002) assumed values for the wind speeds and mass-loss rates to end up with an acceptable configuration from scaling laws. We provide a fully consistent estimate of the wind velocity and maximum mass loss. The velocities we derive in such a way for stars close to the $\Omega \Gamma$-limit are in general in very good agreement with the expansion velocities observed by Weis (2003) for several LBVs, except for $\eta$ Carinae for which they are an order of magnitude too low.

Given the observed geometry of the Homunculus of $\eta$ Carinae, the wind velocity near the equator must be significantly lower than at the pole. In our model shown in Fig. 4 this would correspond to the velocity curve obtained for $0.1 \times$ $10^{-3} M_{\odot} \mathrm{yr}^{-1}$ at the equator. It is quite easy to imagine that the equatorial region would undergo a different effect on its local mass loss from an instability than the pole. A non-radial axisymmetric even oscillation mode, for instance, is just one simple example of an instability that could lead to such an effect. It is clear that our formalism has the potential to lead to very different geometries of the density distribution around a rotating star because we have left the cause of obtaining the ideal velocity gradient at the sonic point unspecified. Several natural large-amplitude phenomena occur at the surface of massive stars (such as stellar oscillations or a complex magnetic field) and may indeed help in the onset of the local mass loss.

We conclude that we have found radiatively-driven wind models below the $\Omega \Gamma$-limit that explain in a natural way the huge currently observed mass-loss rate of $\eta$ Carinae and its spatial distribution. From the point of view of mass loss we would therefore not need any different physical mechanism or a specific eruption to explain the star's nebula and geometry. Our models, however, have too low a wind velocity. We do point out that all other LBVs have expansion velocities that are entirely compatible with the predicted maximum wind velocities of our models.

\section{Discussion}

We determined the maximum mass-loss rates of stars by means of a wind that is lifted out of the potential well by radiation pressure on spectral lines, taking into account rotation. Our calculations were done for a simplistic CAK-type description of the line driving, including the oblateness of the star and the Von Zeipel effect and allowing for the possible presence of an extra 
force or an unspecified instability at the base of the wind (at or below the sonic point) that might increase the mass-loss rate. The maximum mass-loss rates were determined explicitly for 9 stellar models, which are representative for massive stars at different evolutionary stages and not too close to the Eddington limit. All rotating models have higher mass-loss rates at the pole than at the equator, the contrast increasing as the rotation increases. This is in agreement with previous studies of self-regulated winds in the absence of a rotationally induced bistability jump (Pelupessy et al. 2000), as shown by Dwarkadas $\&$ Owocki (2002). For moderately to rapidly rotating stars we find maximum mass-loss rates that are significantly higher than those of non-rotating stars. In particular, all OB stars with nearcritical rotation can have extreme mass loss in the presence of surface instabilities. From comparison of our results with those by Maeder \& Meynet (2000) we conclude that the onset of extreme mass loss occurs at lower rotation rates when allowing an instability to help increase the mass-loss rate or lift the material at the base of the wind.

We provide the maximum loss of angular momentum as function of the stellar parameters and of the rotational velocity for all considered models. It would be worthwhile to compare these angular momentum losses with those used in stellar evolution codes.

We provide a formula to predict the maximum mass-loss rate for stars with a luminosity not too different from $5.0<$ $\log \left(L / L_{\odot}\right)<6$.0. It was derived from multiple regression using the results of our detailed numerical integration of the momentum equation for 9 models. This formula can easily be combined with mass-loss estimates based on multiple scattering for non-rotating stars (Vink et al. 2000, 2001). The use of the formula provided by Maeder \& Meynet (2000) allows one to find the mass lost by massive stars in the presence of rotation without the occurrence of instabilities while our formula leads to the maximum amount of mass that can be lost due to a line-driven wind in a rotating star that undergoes unspecified instabilities which help to reach the optimum velocity gradient at the sonic point.

By means of integration of the momentum equation for the specific case of the LBV $\eta$ Carinae we have found models below the $\Omega \Gamma$-limit that lead to realistic predictions of the huge mass loss observed for this star and of its latitudinal distribution. In general, we are able to explain the combination of high mass loss and low wind velocities observed in LBVs. We were unable, however, to find a model below the $\Omega \Gamma$-limit that predicts the high mass-loss rate in combination with the measured high wind velocity of $\eta$ Carinae itself.

Acknowledgements. The authors are much indebted to prof. André Maeder for his constructive criticism on an earlier draft of the paper, which helped them to make significant improvements to the study. C.A. is grateful to profs. Stan Owocki, Joachim Puls, Rens Waters and drs. Roy van Boekel and Rich Townsend for stimulating discussions.

\section{References}

Aerts, C., \& Lamers, H. J. G. L. M. 2003, A\&A, 403, 625 (Paper I)

Bjorkman, J. E., \& Cassinelli, J. P. 1993, ApJ, 409, 429

Castor, J. I., Abbott, D. C., \& Klein, R. I. 1975, ApJ, 195, 157

Dwarkadas, V. V, \& Owocki, S. P. 2002, ApJ, 581, 1337

Feldmeier, A., \& Shlosman, I. 2002, ApJ, 564, 385

Friend, D. B., \& Abbott, D. C. 1986, ApJ, 311, 701

Gayley, K. G. 2000, ApJ, 529, 1019

Glatzel, W. 1998, A\&A, 339, L5

Hillier, D. J., Davidson, K., Ishibashi, K., \& Gull, T. 2001, ApJ, 553, 837

Lamers, H. J. G. L. M., \& Leitherer, C. 1993, ApJ, 412, 771

Lamers, H. J. G. L. M., Snow, T. P., \& Lindholm, D. M. 1995, ApJ, 455,269

Langer, N. 1998, A\&A, 329, 551

Maeder, A., \& Desjacques, V. 2001, A\&A, 372, L9

Maeder, A., \& Meynet, G. 2000, A\&A, 361, 159

Owocki, S. P., Cranmer, S. R., \& Blondin, J. M. 1994, ApJ, 424, 887

Owocki, S. P., Gayley, K., \& Cranmer, S. R. 1998, in Boulder-Munich II: Properties of hot, luminous stars, ed. I.D. Howarth, ASP Conf. Ser., 131, 237

Pauldrach, A. W. A., Puls, J., \& Kudritzki, R. P. 1986, A\&A, 164, 86

Pelupessy, I., Lamers, H. J. G. L. M., \& Vink, J. S. 2000, A\&A, 359, 695

Poe, C. H., Owocki, S. P., \& Castor, J. I. 1990, ApJ, 358, 199

SAS/ User's Guide 2002, Version 8.2, SAS Institute Inc., Cary, NC

Smith, N., Davidson, K., Gull, T., et al. 2003, ApJ, 586, 432

van Boekel, R., Kervella, P., Schöller, M., et al. 2003, A\&A, 410, L37

Vink, J. S., de Koter, A., \& Lamers, H. J. G. L. M. 2000, A\&A, 362, 295

Vink, J. S., de Koter, A., \& Lamers, H. J. G. L. M. 2001, A\&A, 369, 574

Weis, K. 2003, A\&A, 408, 205 